\title{
Bilateral striatal necrosis, dystonia and optic atrophy in two siblings
}

\author{
V Leuzzi, E Bertini, A M De Negri, M Gallucci, B Garavaglia
}

\begin{abstract}
Two siblings developed a neurological disorder in the first decade characterised by generalised dystonia, hypokinesia, and subacute visual loss. CT and serial MRI examinations showed bilateral lesions of the striatum, mainly in the putamen. The classification of these patients is discussed in relation to infantile bilateral striatal necrosis (IBSN), Leigh's disease, and Leber's optic neuropathy. The literature shows a clinical and aetiopathogenetic overlap between these syndromes. In our cases parental consanguinity and the involvement of a single generation suggest a new clinical condition with autosomal recessive transmission.
\end{abstract}

With the development of non-invasive diagnostic techniques, there has been an increase in reported cases of a progressive dystonic syndrome beginning in childhood and associated with selective lesions of the basal ganglia. ${ }^{12}$ Improvement of CNS investigations has brought new criteria for the differential diagnosis between idiopathic and secondary dystonias. ${ }^{3}$ We report two siblings with dystonia, hypokinesia, symmetrical striatal necrosis, and visual failure. These cases, together with others previously reported, ${ }^{4}$ may represent a new clinical entity.

\section{Patients}

The parents were first cousins. The paternal grandfather had suffered from left hemiparesis, which started at the age of 8 years during an unspecified exanthematic illness. The couple had four children, and the two patients were the product of the second and third pregnancy respectively (the latter was a dizygotic twin pregnancy).

\section{Case 1}

This 20 year old woman had had a normal birth and development. At the age of 6 years she contracted measles. Four days after exanthematic eruption she had transient (a few seconds) amaurosis which disappeared spontaneously without further effects. Six months later she experienced a painless rapid bilateral deterioration in visual acuity. At the age of 7 she began to invert and plantar flex the left foot with dorsiflexion of the big toe when walking; dystonia then slowly progressed to involve her left arm. At the age of 10, she underwent left Achilles tendon and extensor hallucis longus tendon lengthening procedures. At the age of 11, the left dystonic hemiparesis worsened: the hip and the knee were flexed, the foot was equinovarus, the toes plantar flexed. The arm exhibited athetoid postures and movements. Both optic discs were pale. Visual acuity was correctable to VR 1/10, VL 10/10. A CT scan showed an area of low attenuation in the right putamen and in the head of the right caudate nucleus with slight enlargement of the homolateral ventricle.

The patient was re-examined in April 1987 at the age of 18 . In addition to dystonic postures and movements of the left limbs, there was distinct generalised hypokinesia and bradykinesia. Both arms presented high frequency distal postural tremor; there was a plastic rigidity of limbs and tendon reflexes of the left leg were weak. The left kneecap was dislocated upward because of the shortening of the rectus femoris, which showed regular, almost rhythmical (2$3 \mathrm{~Hz}$ ), myoclonic jerks either in the resting condition or during leg movement. She had an external squint. Ophthalmoscopy revealed a bilateral optic atrophy; the vessels were unusually tortuous. On examination, the visual acuity was correctable to VR $3 / 50$, VL $2 / 10$; the study of visual fields (Goldmann) showed an absolute centrocaecal scotoma with a small area of normal central vision on the right eye and an absolute centrocaecal scotoma, multiple absolute paracentral, and peripheral scotomas on the left eye. The right pupil was larger than the left, but light and near reflexes were normal. Visual evoked responses to flash stimuli showed normal voltage and latency; no cortical responses were evoked by patterned stimuli. Brain CT and MRI scans showed bilateral putaminal lesions and involvement of the head of the right caudate nucleus (fig 1). Fasting resting blood lactate concentration was normal, but blood pyruvate and alanine concentrations were elevated $(8.8 \mathrm{mg} / 1$, normal range $3 \cdot 6-5.9 \mathrm{mg} / 1$, and $1061.3 \mu \mathrm{M} / \mathrm{l}$, normal range $273-449 \mu \mathrm{M} / \mathrm{l}$, respectively). She was re-examined in October 1989. Visual acuity had worsened, and she complained of episodes of sudden transient amaurosis for about $\mathbf{1 0}$ minutes several times a day. Neurological, neuro-ophthalmological, and neuroradiological (MRI) examinations were unchanged. Her mental function remained unaffected.

\section{Case 2}

This 11 year old boy had been born preterm (eight months, weight $2550 \mathrm{~g}$ ) after an uncomplicated twin pregnancy and delivery. Growth and development were normal until the age of 7 
Figure 1 MRI examination of case 1 aged 18 years:

left-T1-weighted (IR

1800/400) axial sequence; both putamina (arrows) and head of right caudatus nucleus (curved arrow) show hypointense signal head of left caudatus and anterior third of left putamen are normally represented; right-T1weighted (SE 350/30) coronal scan; arrows indicate bilateral involvement of putamina.

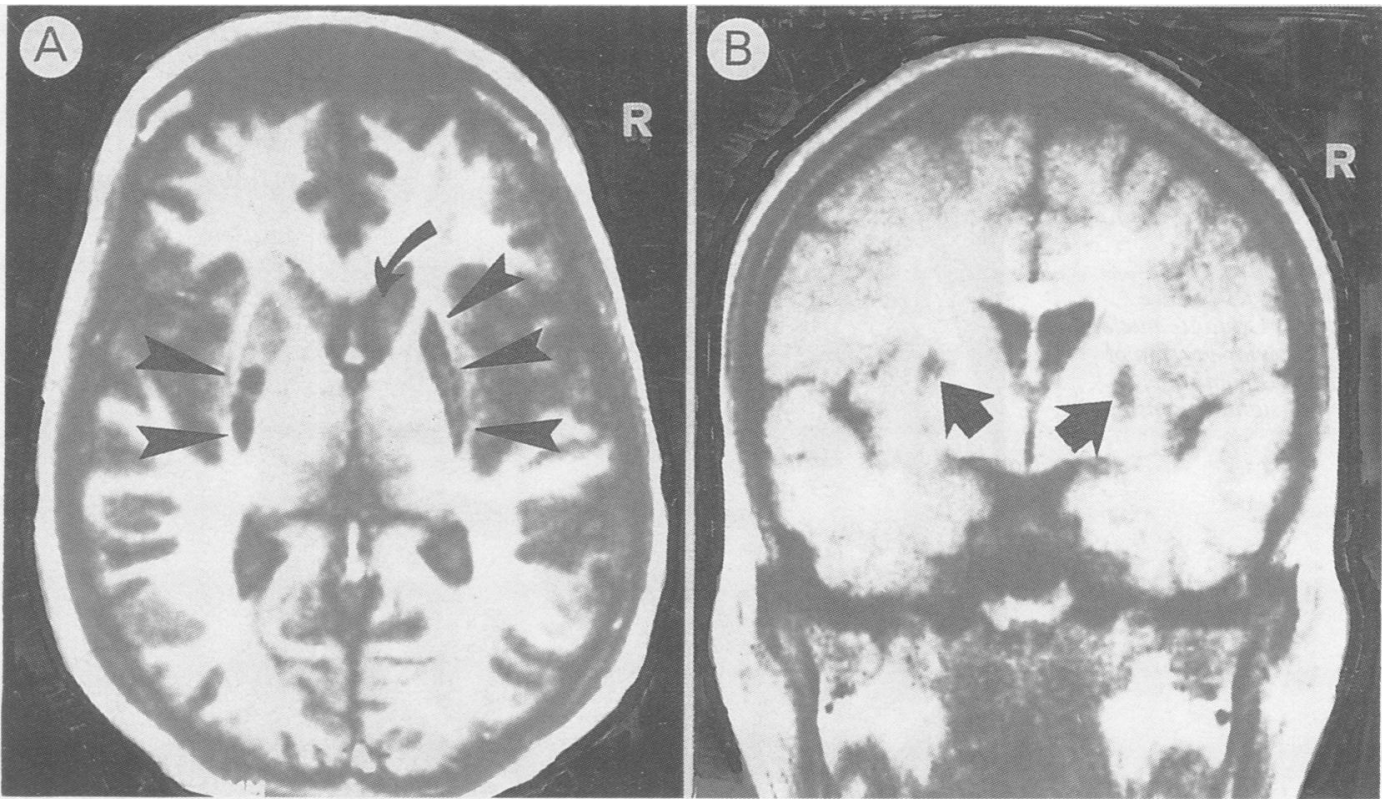

years and 10 months, when his parents noticed that his right arm irregularly adopted bizarre postures (extension and adduction of the arm, pronation of the forearm and hand). Despite abnormal postures, the child could perform skilful movements (such as writing). When 9 years old, he developed an increasingly severe rigidity of the left leg which disturbed walking. The homolateral upper limb was flexed with a clenched fist. He became anorexic and asthenic. On examination (in April 1987), at the age of 9 years and 5 months, he was a pale boy with normal somatic growth, poor muscle development, and dystonic movements and postures of the limbs (left more than right). The most considerable neurological disorders of the arms, however, were hypokinesia and bradykinesia, which were the cause of clumsiness and inadequacy in intentional skilled movements. There was plastic rigidity and mild weakness (left more than right). Tendon reflexes were brisk and polyphasic with bilateral Babinski's signs. Speech was quiet, slow, monotonous, and somewhat slurred. Sensation was intact. Mental function remained unaffected.

Both optic discs showed temporal pallor, and the retinal vessels were narrowed. On examination, the visual acuity was correctable to VR 5/ 10, VL 8/10; perimetry showed a distinct concentric constriction of visual fields and enhanced blind spot. Colour vision was impaired in the red-green region (Ishihara test). Nonsustained horizontal nystagmus to the right was present. Visual evoked responses to flash stimuli showed normal voltage and latency; pattern reversal VEPs showed low amplitude, delayed $\mathrm{P} 2$ component $(140 \mathrm{~ms})$ in $\mathrm{O}_{1}$, and a normal response in $\mathrm{O}_{2}$. A brain CT scan and MRI examination showed bilateral selective lesions of the putamina (fig 2). His fasting resting blood lactate concentration was normal, but on four separate occasions the blood pyruvate concentration was elevated one and a half to three times the normal values. Pyruvate oxidation and respiratory chain function of cultured fibroblasts were normal. Histochemical and ultrastructural studies of left deltoid muscle biopsy specimens showed no abnormalities.

The patient was re-examined when 10 years old. He walked with more difficulty. Intentional movements of the arms were slow and clumsy; his speech was slurred and writing much slower. There was generalised muscular hypotrophy and weakness. Visual acuity was correctable to VR 4/10, VL 6/10. A second brain MRI examination was unchanged. $\mathrm{He}$ underwent clinical assessments every six months until June 1990. Neurological, neuroophthalmological, and neuroradiological (MRI) examinations revealed no change from his previous state. He had no intellectual impairment or learning difficulties. His somatic development, however, was arrested (height below $10 \%$ and weight below $3 \%$ ).

The analysis of mitochondrial DNA obtained by PCR-amplification on total DNA from muscle specimens showed a normal Sfa NI digestion of the $316 \mathrm{bp}$ PCR product, that includes the nt 11778 mutation associated with Leber's hereditary optic neuropathy ${ }^{5}$ (Dr C Gellera, Istituto Neurologico "Besta" Milano). In both patients values were normal for the following analyses: standard blood and urinary studies, VDRL, serum copper and caeruloplasmin, 24 hour urinary copper, serum lipid, serum protein electrophoresis, plasma and urinary aminoacid chromatography, urinary mucopolysaccharides, urinary organic acids, urinary arylsulfatase- $\mathrm{A}$, and an assay of lysosomal enzymes in white blood cells and fibroblasts in case 2. EMG and nerve conduction studies, EEG, BAEP, SEP, ERG, and slit lamp examination for Kayser-Fleischer ring were also normal. The twin of case 2 has been surveyed for four years. He is an intelligent, normal child with no motor or neuro-ophthalmological disability. Three brain MRI examinations performed at intervals of six months gave normal results. 
Figure 2 MRI

examination of case 2 aged 10 years. Selective involvement of both putamina (arrows), appear hypointense in Ti-weighted

(IR 1800/400) (left) and hyperintense in

T2-weighted ( $S E$

1800/120) sequence

(right). Caudate nucle

and anterior portion of

right putamen show

normal signal intensity.
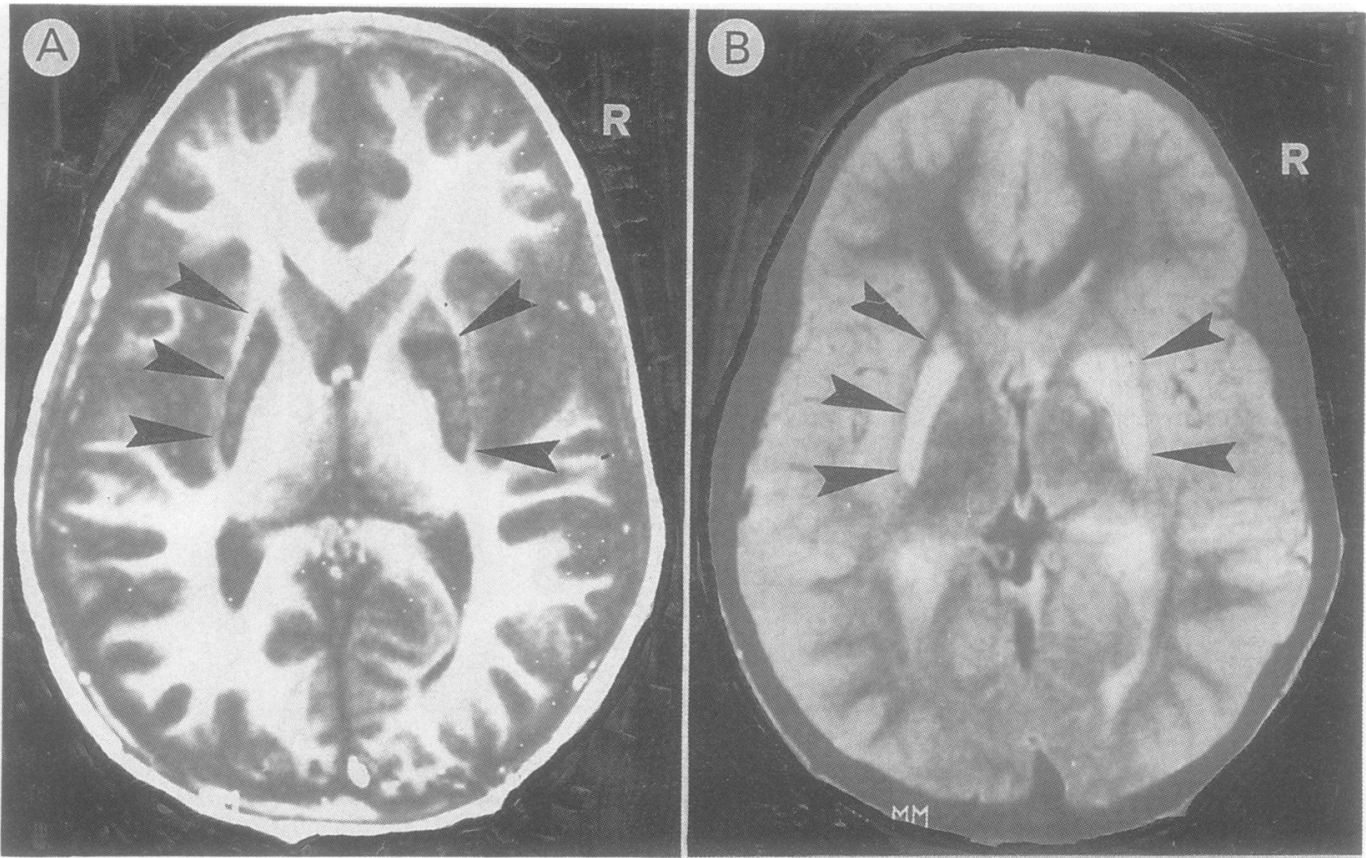

\section{Discussion}

The two siblings reported here show an uncommon clinical disorder characterised by dystonic movements and postures associated with generalised hypokinesia, subacute visual loss of varying degrees, and bilateral and selective lesions of the striatum detected by neuroradiological techniques (CT and MRI). Mental deterioration was absent. We suggest that three clinico-pathological conditions should be considered; infantile bilateral striatal necrosis (IBSN), Leigh's disease, and Leber's optic neuropathy (LHON). With the term of infantile bilateral striatal necrosis, Friede ${ }^{6}$ defined a group of infantile encephalopathies in which the main pathological finding was bilateral, symmetrical, spongy degeneration of putamina, caudate nuclei, and, less commonly, pallidum. Modern imaging techniques such as brain CT and MRI can detect basal ganglia abnormalities. ${ }^{7}$ Though they cannot substitute neuropathological examination, they do enable new cases of IBSN to be observed in vivo, and serial studies can assess the evolution of the disease.

Cases reviewed by Friede, as well as those more recently described, ${ }^{48-13}$ are clinically heterogeneous. Age at onset varies from infancy to adulthood; some cases present a progressive disorder, others an acute or subacute neurological disorder, sometimes preceded by an acute febrile illness. Clinical features, with various combinations, include dystonia, hyperkinesia, spasticity, bilateral optic atrophy, abnormal eye movements, seizures, mental retardation or mental deterioration, disturbed behaviour, and failure to thrive. The disorder has a poor prognosis: death usually occurs, but at intervals from a few weeks to some years after onset of symptoms. Cases with complete or partial remission, however, have also been reported..$^{10}$ All previously reported familial cases were in one generation or the same sibship, ${ }^{411}{ }^{14-17}$ suggest- ing autosomal recessive transmission. The family described by Bargeton-Farkas et al $^{15}$ is the only published example of parental consanguinity we know of.

Confining the discussion to slowly progressive cases, those described by Miyoshi et $a^{16}$ and Pebenito et al ${ }^{11}$ differ from ours in that mental retardation was prominent in the former and visual function was unaffected in all. Both clinical and neuroradiological findings of our patients are similar to the clinical observations of Marsden et al, ${ }^{4}$ who described seven patients in two families. The patients exhibited a slowly progressive generalised dystonic syndrome, which was variably associated with subacute visual loss and striking bilateral symmetrical lucencies on CT, particularly affecting the putamen. The patients showed no obvious mental deterioration. In one case the visual deficit was isolated and in another a diagnosis of Leigh's disease was suggested. Symmetrical areas of low attenuation in the basal ganglia on CT scan, dystonic movements, optic atrophy, and increase of blood pyruvate and alanine are often observed in patients with Leigh's disease. ${ }^{18-21}$ This constitutes a heterogeneous group of disorders associated with a disturbance in mitochondrial energy metabolism. ${ }^{18}$ The clinical features are variable and are related to the multiplicity and site of the neuropathological lesions. ${ }^{1822}$ The coexistence of multiple neurological deficits is also found in late onset cases which usually progress more slowly. ${ }^{23} 24$ Recently, Van Erven et a ${ }^{25}$ reported three sporadic patients with a slowly progressive condition like Leigh's disease in which hypokinesia and rigidity were the most prominent neurological disorders. Nevertheless, other signs and symptoms in these cases suggest the multi-systemic aspect typical of Leigh's disease: deterioration during intercurrent infections followed by a slow recovery, exercise intolerance, poor somatic growth, abrupt changes in respiratory and cardiac rates, 
strabismus, nystagmus, dysarthria, ataxia, tremor, and dysmetria.

Neuroradiological data derived from MRI, particularly if serial studies are performed, offer a valuable tool for differentiating in vivo selective lesions of the striate nuclei, as in IBSN, from the multifocal involvement of the brain seen in Leigh's disease. ${ }^{26-29}$ In the absence of a neuropathological report, we believe that Leigh's disease is unlikely in our patients as no damage was present outside the striatum (as detected repeatedly by CT and MRI) and no clinical signs of brainstem, cerebellar, and peripheral nerve involvement were ever noted. Moreover, we did not find any pyruvate oxidation defects and respiratory chain abnormalities in fibroblasts of case 2 .

In our patients, subacute painless visual failure suggests the diagnosis of Leber's optic neuropathy (LHON). This is a maternally inherited form of optic nerve atrophy associated with neurological and psychiatric symptoms in a high percentage of cases, both in affected patients and in their collaterals. ${ }^{3031} \mathrm{~A}$ few reports exist in which LHON is associated with IBSN. Novotny et al ${ }^{32}$ studied a single large pedigree ( 79 members in five generations) of patients affected by Leber's disease. Eight members had neuroretinopathy, 14 had a progressive, generalised dystonia attributed to striatal degeneration, but only one had both disorders. In six out of 14 neurologically affected patients CT examination demonstrated low-density in the putamen as the earliest finding. Caudate nuclei were affected later, and one subject had low density lesions in the centromedian nucleus region of both thalami. Recently, Wallace et $a l^{5}$ and Singh et $a l^{33}$ identified a mitochondrial DNA point mutation (at nt 11778) that correlated with Leber's disease in nine out of 11 tested pedigrees. The authors did not find the same mutation, however, in cases of LHON plus IBSN, which may be the result of the presence of a mixture of mutant and normal mitochondrial DNA sequences (heteroplasmy). ${ }^{34}$

Our two patients were not seen in the acute phase of the optic neuropathy, when the peripapillary microangiopathy gives a pathognomonic appearance of Leber's disease. ${ }^{35}$ The clinical picture, some ophthalmoscopic findings (arterioral attenuation and persistence of tortuous vessels in case 1), and visual field deficits may suggest this disease. Although the restriction-fragment-length polymorphism negativity after digestion of mitochondrial DNA with Sfa NI enzyme might accord with the hypothesis of different genetic origins of classical LHON and LHON associated with IBSN, the parental consanguinity and the apparent involvement of a single generation suggest a new clinical condition with an autosomal recessive transmission.

1 Aicardi J, Gordon N, Hagberg B. Holes in the brain. Devel Med Child Neurol 1985;27:249-52.
2 Leuzzi V, Favatà I, Seri S. Bilateral striatal lesions. Devel Med Child Neurol 1988;30:252-7.

3 Marsden CD. Investigation of dystonia. Adv Neurol 1988; 50:35-44.

4 Marsden CD, Lang AE, Quinn NP, McDonald WI, Abdallat A, Nimri S. Familial dystonia and visual failure with striatal luciencies. J Neurol Neurosurg Psychiatry 1986;49:500-9.

5 Wallace DC, Singh G, Lott MT, et al. Mitochondrial DNA mutation associated with Leber's hereditary optic neuropathy. Science 1988;242:1427-30.

6 Friede RL. Developmental neuropathology. Wien: Springer, 1975:88-9.

7 Rutledge JN, Hilal SK, Silver AJ, Defendini R, Fhan S. Magnetic resonance imaging of dystonic states. $A d v$ Neurol 1988;50:265-75.

8 Erdohazi M, Marshall P. Striatal degeneration in childhood. Arch Dis Child 1979;54:85-91.

9 Röyttä M, Olsson I, Sourander P, Svendsen P. Infantile bilateral striatal necrosis. Acta Neuropath 1981;55: 97-103.

10 Goutières F, Aicardi J. Acute neurological dysfunction associated with destructive lesions of the basal ganglia in children. Ann Neurol 1982;12:328-32.

11 Pebenito R, Ferretti C, Chaudary RR, Wooddrow PK. Idiopathic torsion dystonia with lesions of the basal ganglia. Clin Pediatr 1984;23:232-5.

12 Mito T, Tanaka T, Becker LE, Takashima S, Tanaka J. Infantile bilateral striatal necrosis. Clinicopathological Infantile bilateral striatal necrosis. Clinicop
classification. Arch Neurol 1986;43:677-80.

13 Berkovic SF, Karpati G, Carpenter S, Lang AE. Progressive dystonia with bilateral putaminal hypodensities. Arch Neurol 1987;44:1184-7.

14 Paterson D, Carmichael EA. A form of familial cerebral degeneration chiefly affecting the lenticular nucleus. Brain 1924;47:207-31.

15 Bargeton-Farkas E, Cochard AM, Brissaud HE, Robain O, Le Balle JC. Encéphalopathie infantile familiale avec nécrose bilatérale et symétrique des corps Striés. J Neurol Sci 1964;1:429-45.

16 Miyoshi K, Matsuoka T, Mizushima S. Familial holotopistic striatal necrosis. Acta Neuropath 1969;13:240-9.

17 Roessmann U, Schwartz JF. Familial striatal degeneration. Arch Neurol 1973;29:314-7.

18 Van Erven PMM, Cillessen JPM, EekhoffEMW, et al. Leigh syndrome, a mitochondrial encephalo(myo)pathy. Clin Neurol Neurosurg 1987;89:217-30.

19 Hall K, Gardner-Medwin D. CT scan appearances in Leigh's disease (subacute necrotizing encephalomyelopathy). Neuroradiology 1978;16:48-50.

20 Schwartz WJ, Hutchinson HT, Berg BO. Computerized tomography in subacute necrotizing encephalomyelopathy (Leigh Disease). Ann Neurol 1981;10:268-71.

21 Campistol J, Fernandez Alvarez E, Cusi V. CT appearance in subacute necrotising encephalomyelopathy. Dev Med Child Neurol 1984;26:519-21.

22 Montpetit VJA, Andermann F, Carpenter S, Fawcett JS, Zborowska-Sluis D, Giderson HR. Subacute necrotizing encephalomyelopathy. A review and a study of two families. Brain 1971;94:1-30.

23 Whetsell WO, Plaitakis A. Leigh's disease in an adult with evidence of "inhibitor factor" in family members. Ann Neurol 1978;3:519-24
Nence of "inhibitor

24 Plaitakis A, Whetsell WO Jr, Cooper JR, Melvin DY. Chronic Leigh disease: a genetic and biochemical study. Ann Neurol 1980;7:304-10.

25 Van Erven PMM, Renier WO, Gabreels FJM, Thijssen HOM, Ruitenbeek W, Horstink MWIM. Hypokinesia and rigidity as clinical manifestations of mitochondrial encephalomyopathy: report of three cases. Dev Med Child Neurol 1989;31:81-91.

26 Koch TK, Yee MHC, Hutchinson HT, Berg BO. Magnetic resonance imaging in subacute necrotizing encephalomyelopathy (Leigh's disease). Ann Neurol 1986;19:605-7.

27 Davis PC, Hoffman JC, Braun IF, Ahmann P, Krawiecki N. MR of Leigh's disease (subacute necrotizing encephalomyelopathy). Am J Neuroradiol 1987;8:71-5.

28 Kissel JT, Kolkin S, Chakeres D, Boesel C, Weiss K Magnetic resonance imaging in a case of autopsy-proved Magnetic resonance imaging in a case of autopsy-proved
adult subacute necrotizing encephalomyelopathy (Leigh's adult subacute necrotizing encephalo
disease). Arch Neurol 1987;44:563-6.

29 Medina L, Chi TL, De Vivo DC, Hilal SK. MR findings in patients with subacute necrotizing encephalomyelopathy Leigh syndrome): correlation with biochemical defect. Am J Neuroradiol 1990;11:379-84.

30 Bruyn GW, Went LN. A sex-linked heredo-degenerative neurological disorder, associated with Leber's optic atrophy. Part 1 (clinical studies). J Neurol Sci 1964;1:59-80.

31 Palan A, Stehouwer A, Went LN. Studies on Leber's optic neuropathy III. Doc Ophthalmol 1989;71:77-92.

32 Novorny EJ, Singh G, Wallace DC, et al. Leber's disease and dystonia: a mitochondrial disease. Neurology 1986;36: $1053-60$.

33 Singh G, Lott MT, Wallace DC. A mitochondrial DNA mutation as a cause of Leber's hereditary optic mutation as a cause of Leber's hered

34 Wallace DC. Mitochondrial DNA mutation and neuromuscular disease. Trends Genet 1989;5:9-13.

35 Nikoskelainen E, Sogg RL, Rosenthal AR, Friberg TR Dorfman LS. The early phase in Leber's hereditary optic atrophy. Arch Ophthalmol 1977;95:969-78. 